\title{
Induced changes in the consumption of coffee alter ad libitum dietary intake and physical activity level
}

\author{
Annhild Mosd $\varnothing 1^{1 *}$, Benedicte Christensen ${ }^{2}$, Lars Retterst $\varnothing 1^{3}$ and Dag S. Thelle $e^{4,5}$ \\ ${ }^{1}$ Section for Preventive Medicine and Epidemiology, Department of General Practice and Community Medicine, University \\ of Oslo, Norway \\ ${ }^{2}$ Department of Medical Genetics, Ullevål University Hospital, Oslo, Norway \\ ${ }^{3}$ Institute of Medical Genetics, University of Oslo, Norway \\ ${ }^{4}$ Department of Epidemiology and Health Surveillance, Ullevål University Hospital, Oslo, Norway \\ ${ }^{5}$ Institute of Cardiovascular Diseases, Sahlgrenska University Hospital, Göteborg, Norway
}

(Received 21 June 2000 - Revised 21 September 2001 - Accepted 5 November 2001)

\begin{abstract}
Dietary trials with subjects on a freely selected diet may be affected by unwanted behavioural changes. Few studies, if any, have examined changes in coffee consumption and possible concomitant changes in diet and health-related habits. The aim of the present study was to examine whether induced changes in coffee consumption lead to changes in food habits and leisure-time physical activity. Healthy, non-smoking coffee-drinkers $(n 214)$ were asked to change their coffee habits in a controlled clinical trial on the metabolic effects of coffee. The participants were asked to maintain their usual dietary habits. Self-perceived changes in diet and physical activity during the 6-week intervention period were assessed at the end. In the analyses, the participants were rearranged into groups reflecting the difference in coffee intake during the trial as compared with habitual intake. Associations with changes in food intake or physical activity were analysed by Spearman rank correlation. Changes in intake of 'chocolate, sweets' $(r$ 0.179, $P<0.05)$, 'cakes, sweet biscuits, pastry' $(r 0.306, P<0.001)$, and 'jam' ( $r$ $0 \cdot 198, P<0.05)$ showed positive associations with change in coffee intake during the trial. Negative associations were found for 'dishes with fish' $(r-0.204, P<0.01)$ and many of the drinks as well as with physical activity $(r-0 \cdot 164, P<0 \cdot 05)$. Induced changes in coffee intake seem to alter ad libitum intake of several foods. The recognized associations between health behaviours may have physiological explanations.
\end{abstract}

Coffee: Diet: Food preferences: Physical activity

Dietary trials with subjects on a freely selected diet frequently create interpretation problems due to the need for a rather constant intake of energy and fluid. Any induced change in the sources of these two variables is likely to be compensated for by changes in other sources. If these changes are systematic, they must be accounted for, unless the replacements are inert with regard to the outcome variables. This problem is highly relevant in studies on the metabolic effects of coffee. Several crosssectional studies describe a more atherogenic diet among high consumers of coffee compared with coffee abstainers (Haffner et al. 1985; Jacobsen \& Thelle 1987; Solvoll et al. 1989; Puccio et al. 1990). Other factors such as smoking and physical inactivity often accompany this picture. These findings can either be due to high coffee consumers being generally uninterested in or unaware of a healthy lifestyle, or that consumption of coffee, through an unknown mechanism, can alter preference, appetite or craving for particular food groups. Only the second explanation is likely to be relevant when assessing the results from a controlled clinical trial.

While the metabolic effects of coffee have been given quite extensive attention, we are not aware of any studies examining changes in coffee consumption and possible concomitant changes in dietary habits in a trial setting. The results from the present study emerge from a randomized clinical trial where the objective was to examine the effect of coffee on the level of homocysteine in plasma and total cholesterol in serum of free-living subjects (Christensen et al. 2001). This gave us the opportunity to assess whether 
induced changes in coffee consumption among regular coffee drinkers altered the participants' consumption of selected foods. The food categories were sorted in two rough groups containing foods considered to give either an atherogenic or a non-atherogenic diet when eaten in significant quantities. Based on the previous cross-sectional studies reporting associations between coffee intake and particular food groups, we expected positive associations between changes in coffee intake and atherogenic food categories, and negative associations with non-atherogenic categories. Our hypothesis was that increased or decreased consumption of coffee would lead to systematic changes in food habits during a 6-week intervention period. The initial food habits in different strata of habitual coffee consumption and the changes in leisure-time physical activity during the trial are also described.

\section{Subjects and methods}

The study was organized as a prospective, unblinded controlled trial with three different groups that were to consume either no coffee, one to three cups of coffee per d, or four or more cups of coffee per d for 6 consecutive weeks. Design and sample size were chosen based on the expected changes in homocysteine between the groups (Christensen et al. 2001).

The participants were recruited through advertisements in the largest newspaper in Oslo, Norway. Inclusion criteria were: age range 30-69 years, a history of daily consumption of coffee for at least 5 years, and no daily tobacco smoking for the past 6 months. Exclusion criteria were clinically recognized chronic diseases such as cardiovascular diseases, cancer, renal disorders, liver disease or diabetes mellitus, the use of anti-epileptic or cholesterol-lowering drugs, and recognized vitamin deficiency in the past. After recruitment, the participants were randomly allocated to one of the three groups for a 6 -week period. The coffee-consuming groups were permitted to drink the type of coffee that they were accustomed to. Altogether, $97 \%$ of the participants reported that they were regular consumers of caffeinated filtered coffee. Participants were asked to stick to their usual diet and lifestyle. Height and weight were recorded at the beginning and the end of the trial. The first participants entered the trial in April 1999 and the last finished late in June 1999.

At the start of the intervention period, the participants were asked to fill in a Norwegian semi-quantitative foodfrequency questionnaire. This food-frequency questionnaire, which includes approximately 180 questions about 149 food categories, was designed to cover the whole diet. It has previously been validated against $14 \mathrm{~d}$ weighed records (Nes et al. 1992; Andersen et al. 1996, 1999), verylong-chain $n$-3 fatty acids in plasma phospholipids (Andersen et al. 1996, 1999) and $\alpha$-tocopherol in adipose tissue and serum (Andersen et al. 1996). These studies showed that the questionnaire could be used for assessing group intakes of energy and a wide range of nutrients. The daily intake of energy and nutrients was computed by using a food database and software system developed at the Institute of Nutrition Research, University of Oslo. The food database is mainly based on the official Norwegian food composition tables.

During the final medical examination, information was gathered on behavioural changes that might affect the outcome of the main study, including changes in diet and physical activity. The participants completed a second questionnaire regarding their self-perceived changes in intake of twelve food categories and seven categories of drinks, described as 'increased intake', 'unchanged intake' or 'decreased intake', and changes in physical activity pattern during the intervention period. They also reported compliance with the intervention and side effects.

For the present study, the participants were rearranged into four groups to reflect the direction of changes in coffee intake rather than the absolute coffee intake level. Changes in coffee consumption were recorded as the difference between the habitual intake (average number of cups per d) and the coffee consumption during the trial. All the participants allocated to the coffee-abstention group were kept together in one group. The remaining participants were divided into three groups reflecting whether they had decreased their coffee intake to a lower level during the intervention, had unchanged coffee intake $( \pm 0.5$ cup per d), or increased coffee intake. The groups were ordered on an ordinal scale from 1 to 4 (1, abstainers; 2, decreased intake; 3 , unchanged intake; 4 , increased intake). The selfperceived changes in intake were coded along an ordinal scale (1, decreased intake; 2, unchanged intake; 3, increased intake). At baseline, the average intake of coffee was higher among the men than the women. To avoid different proportions of men and women in the baseline strata, the material was split by gender before analysing the diet in relation to habitual coffee consumption. Trends in dietary composition according to strata of habitual coffee consumption were analysed by multiple linear regression. Some of the variables were not normally distributed and were therefore ln-transformed before the analyses. The final outcome did not differ when compared to the same analyses on the original variables.

Difference in habitual coffee intake and age in the coffee change groups were tested with analyses of variance. The Pearson $\chi^{2}$ test was used for comparing the proportion of each gender and the changes in physical activity in the groups. The associations between changes in coffee intake and food intake were analysed with Spearman rank correlation (ordinal by ordinal). Statistical significance was considered at $P$ values $<0 \cdot 05$. The statistical package SPSS (SPSS for Windows, 1997; SPSS Inc., Chicago, IL, USA) was used for all the analyses.

\section{Results}

Two of the 214 healthy persons recruited did not turn up to the appointed visits and four decided to withdraw during the trial. Of the remaining 208 persons, ninety-five men and 111 women completed the food-frequency questionnaire and ninety-five men and 110 women completed the questionnaire regarding self-perceived changes in food intake. Mean age was 53.7 (SD 11.3) years for the men and $50 \cdot 1$ (SD 11.3) years for the women.

The intakes of energy and some selected nutrients in 
Table 1. Intakes of energy and selected nutrients at baseline in four quartiles of habitual coffee consumption among men* (Mean values and standard deviations)

\begin{tabular}{|c|c|c|c|c|c|c|c|c|c|c|c|c|}
\hline & & & \multicolumn{8}{|c|}{ Quartiles of coffee intake } & \multirow[b]{3}{*}{$P+$} & \multirow[b]{3}{*}{$P \ddagger$} \\
\hline & \multicolumn{2}{|c|}{ All men ( $n$ 95) } & \multicolumn{2}{|c|}{$1(n 28)$} & \multicolumn{2}{|c|}{$2(n 16)$} & \multicolumn{2}{|c|}{$3(n 30)$} & \multicolumn{2}{|c|}{$4(n 21)$} & & \\
\hline & Mean & SD & Mean & SD & Mean & SD & Mean & SD & Mean & SD & & \\
\hline Coffee intake (cups per d) & $5 \cdot 3$ & $2 \cdot 6$ & $2 \cdot 7$ & 0.5 & $4 \cdot 0$ & 0.2 & $6 \cdot 0$ & 0.7 & $9 \cdot 2$ & 1.9 & - & - \\
\hline Energy intake $(\mathrm{MJ} / \mathrm{d})$ & $10 \cdot 2$ & 3.0 & 9.4 & $2 \cdot 9$ & $10 \cdot 1$ & $2 \cdot 4$ & $10 \cdot 0$ & $2 \cdot 6$ & 11.9 & 3.8 & 0.01 & 0.01 \\
\hline \multicolumn{13}{|l|}{ Energy distribution (\% energy) } \\
\hline Fat & $30 \cdot 6$ & 5.5 & $28 \cdot 6$ & $5 \cdot 1$ & $30 \cdot 0$ & $5 \cdot 3$ & $31 \cdot 6$ & $5 \cdot 6$ & $32 \cdot 3$ & $5 \cdot 3$ & 0.01 & 0.01 \\
\hline Protein & $15 \cdot 8$ & $2 \cdot 0$ & $16 \cdot 3$ & $2 \cdot 5$ & $15 \cdot 4$ & $1 \cdot 7$ & $15 \cdot 7$ & 1.9 & $15 \cdot 5$ & 1.9 & 0.24 & 0.25 \\
\hline Carbohydrate & $49 \cdot 9$ & $6 \cdot 1$ & $51 \cdot 2$ & $6 \cdot 1$ & $50 \cdot 0$ & $5 \cdot 8$ & $49 \cdot 0$ & $5 \cdot 5$ & $49 \cdot 5$ & $7 \cdot 2$ & 0.23 & 0.21 \\
\hline Alcohol & 3.7 & $4 \cdot 3$ & 3.9 & 4.5 & 4.6 & 5.5 & 3.7 & $4 \cdot 6$ & $2 \cdot 6$ & $2 \cdot 4$ & 0.28 & 0.28 \\
\hline \multicolumn{13}{|l|}{ Nutrient intake } \\
\hline$\beta$-Carotene $(\mu \mathrm{g} / \mathrm{d}$ per $10 \mathrm{MJ})$ & 4610 & 3630 & 4940 & 4080 & 4730 & 4910 & 4420 & 2510 & 4380 & 3450 & $0.70^{\S}$ & $0.70^{\S}$ \\
\hline Vitamin C (mg/d per $10 \mathrm{MJ})$ & 151 & 72 & 188 & 89 & 127 & 35 & 139 & 70 & 139 & 57 & $0.03^{\S}$ & $0.03^{\S}$ \\
\hline Fibre $(\mathrm{g} / \mathrm{d}$ per $10 \mathrm{MJ})$ & $26 \cdot 1$ & 6.5 & $27 \cdot 7$ & 7.5 & $25 \cdot 3$ & 6.4 & $25 \cdot 2$ & 6.5 & $26 \cdot 1$ & $5 \cdot 3$ & $0.37^{\S}$ & $0.39^{\S}$ \\
\hline
\end{tabular}

* For details of subjects and procedures, see p. 262.

$\dagger$ Test for trend.

tond, adjusted for age.

§Analyses were based on In-transformed data.

Table 2. Intakes of energy and selected nutrients at baseline in four quartiles of habitual coffee consumption among women (Mean values and standard deviations)

\begin{tabular}{|c|c|c|c|c|c|c|c|c|c|c|c|c|}
\hline & & & \multicolumn{8}{|c|}{ Quartiles of coffee intake } & \multirow[b]{3}{*}{$P \dagger$} & \multirow[b]{3}{*}{$P \neq$} \\
\hline & \multicolumn{2}{|c|}{ All women $(n 111)$} & \multicolumn{2}{|c|}{$1(n 29)$} & \multicolumn{2}{|c|}{$2(n 29)$} & \multicolumn{2}{|c|}{$3(n 20)$} & \multicolumn{2}{|c|}{$4(n 33)$} & & \\
\hline & Mean & SD & Mean & SD & Mean & SD & Mean & SD & Mean & SD & & \\
\hline Coffee intake (cups per d) & 4.5 & $2 \cdot 4$ & $2 \cdot 1$ & 0.5 & $3 \cdot 3$ & 0.4 & $5 \cdot 1$ & 0.4 & $7 \cdot 2$ & $2 \cdot 1$ & - & - \\
\hline Energy intake $(\mathrm{MJ} / \mathrm{d})$ & 8.0 & $2 \cdot 3$ & $8 \cdot 2$ & $2 \cdot 0$ & $7 \cdot 7$ & $2 \cdot 1$ & 7.8 & $2 \cdot 2$ & 8.4 & 2.6 & 0.60 & 0.54 \\
\hline \multicolumn{13}{|l|}{ Energy distribution (\% energy) } \\
\hline Fat & $30 \cdot 6$ & 4.7 & $30 \cdot 2$ & $5 \cdot 3$ & $31 \cdot 1$ & 3.8 & $30 \cdot 8$ & 5.5 & 30.5 & 4.6 & 0.88 & 0.85 \\
\hline Protein & $15 \cdot 8$ & $2 \cdot 0$ & $15 \cdot 8$ & $2 \cdot 0$ & $15 \cdot 6$ & 1.9 & $16 \cdot 3$ & $2 \cdot 7$ & $15 \cdot 6$ & 1.7 & 0.96 & 0.88 \\
\hline Carbohydrate & 51.4 & 4.7 & $51 \cdot 8$ & 5.5 & $51 \cdot 2$ & $3 \cdot 1$ & $50 \cdot 4$ & $6 \cdot 3$ & 51.7 & 4.2 & 0.84 & 0.86 \\
\hline Alcohol & $2 \cdot 2$ & 1.9 & $2 \cdot 2$ & $2 \cdot 0$ & $2 \cdot 1$ & 1.5 & $2 \cdot 4$ & $2 \cdot 6$ & $2 \cdot 2$ & 1.9 & 0.92 & 0.96 \\
\hline \multicolumn{13}{|l|}{ Nutrient intake } \\
\hline$\beta$-Carotene ( $\mu \mathrm{g} / \mathrm{d}$ per $10 \mathrm{MJ})$ & 6050 & 3860 & 7710 & 4790 & 5160 & 3330 & 5240 & 3110 & 5870 & 3490 & $0.27^{\S}$ & $0.22^{\S}$ \\
\hline Vitamin C (mg/d per $10 \mathrm{MJ})$ & 199 & 125 & 236 & 131 & 182 & 129 & 173 & 70 & 199 & 140 & $0.27^{\S}$ & $0.22^{\S}$ \\
\hline Fibre (g/d per $10 \mathrm{MJ})$ & $28 \cdot 3$ & $7 \cdot 1$ & 30.4 & $7 \cdot 3$ & $26 \cdot 6$ & $6 \cdot 0$ & $27 \cdot 1$ & $7 \cdot 1$ & $28 \cdot 7$ & $7 \cdot 7$ & $0.51^{\S}$ & $0.38^{\S}$ \\
\hline
\end{tabular}

${ }^{*}$ For details of subjects and procedures, see p. 262.

$\dagger$ Test for trend.

$\ddagger$ Test for trend, adjusted for age.

$\S$ Analyses were based on In-transformed data. 
Table 3. Distribution of variables in the four participant groups $\ddagger$ (Mean values and standard deviations)

\begin{tabular}{|c|c|c|c|c|c|c|c|c|c|c|}
\hline & \multicolumn{2}{|c|}{ All $(n 205)$} & \multicolumn{2}{|c|}{$\begin{array}{l}\text { Abstainers } \\
\text { (n 69) }\end{array}$} & \multicolumn{2}{|c|}{$\begin{array}{l}\text { Decreased } \\
\quad(n 46)\end{array}$} & \multicolumn{2}{|c|}{$\begin{array}{l}\text { Unchanged } \\
\qquad(n 50)\end{array}$} & \multicolumn{2}{|c|}{$\begin{array}{l}\text { Increased } \\
\quad(n 40)\end{array}$} \\
\hline & Mean & SD & Mean & SD & Mean & SD & Mean & SD & Mean & SD \\
\hline Habitual coffee intake (cups per $d)^{\star \star \star}$ & $4 \cdot 8$ & $2 \cdot 5$ & $5 \cdot 1$ & $2 \cdot 5$ & $6 \cdot 4$ & $2 \cdot 3$ & $4 \cdot 7$ & $2 \cdot 4$ & $2 \cdot 7$ & $1 \cdot 1$ \\
\hline Coffee intake during the trial (cups per d) & $2 \cdot 8$ & 2.5 & 0.0 & & 2.9 & $1 \cdot 0$ & $4 \cdot 7$ & $2 \cdot 4$ & 4.9 & $1 \cdot 3$ \\
\hline Change in coffee intake (during - before) & $-2 \cdot 0$ & 3.5 & $-5 \cdot 1$ & 2.5 & -3.6 & $2 \cdot 3$ & -0.01 & $0 \cdot 2$ & $2 \cdot 3$ & $1 \cdot 1$ \\
\hline Age (years) $\dagger$ & $51 \cdot 7$ & $11 \cdot 5$ & $51 \cdot 8$ & $10 \cdot 6$ & $52 \cdot 2$ & $11 \cdot 2$ & $50 \cdot 3$ & $13 \cdot 2$ & $52 \cdot 6$ & $11 \cdot 3$ \\
\hline Proportion women (\%)* & $53 \cdot 4$ & & $65 \cdot 2$ & & $46 \cdot 7$ & & $39 \cdot 2$ & & $58 \cdot 5$ & \\
\hline
\end{tabular}

Mean values were significantly different between the groups: ${ }^{\star} P<0.05$, ${ }^{\star \star \star} P<0.001$.

$\dagger$ There were no significant differences between the groups.

$\ddagger$ For details of subjects and procedures, see p. 262.

quartiles of habitual coffee consumption are shown in Tables 1 and 2. $\beta$-Carotene, vitamin $\mathrm{C}$ and fibre were chosen as nutrients representing the intake of vegetables, fruits and cereals. For men, the mean coffee intake was 2.7 cups per $\mathrm{d}$ in the lowest strata and 9.2 cups per $\mathrm{d}$ in the highest. The corresponding values were $2 \cdot 1$ and $7 \cdot 2$ cups per $\mathrm{d}$ for the women respectively.

Among the men, both the mean energy intake and the \% energy from fat showed a significant increasing trend over quartiles of coffee intake. There was an opposite trend in intake of vitamin $\mathrm{C}$. The trends were not affected by adjustment for age. None of the other nutrients revealed significant associations with habitual coffee intake. Analyses of the women's diet did not show any significant associations between habitual coffee intake and the selected nutrients.

To determine whether the participants changed their food habits during the trial, the sample was rearranged into four groups reflecting the difference in coffee intake during the trial as compared with habitual intake. Characteristics of the participants in each group are shown in Table 3. Due to the study design, there was an overall average reduction in coffee consumption by 2 cups per $\mathrm{d}$. The proportion of men and women in the groups were not the same. However, the age distribution was not significantly different between the groups, either when the groups were analysed with the genders together or when men and women were analysed separately (results not shown).

Table 4 presents associations between changes in coffee consumption and self-evaluated changes in intake of food and drink categories during the trial. Three of the six categories classified as atherogenic showed significant positive associations with change in coffee intake. Both the categories 'chocolate and sweets' and 'cakes, sweet

Table 4. Associations between changes in coffee consumption and self-evaluated changes in intake of food categories during the trial†‡

\begin{tabular}{|c|c|c|c|}
\hline & All $(n 205)(r) \S$ & Men $(n 95)(r)$ & Women $(n 111)(r)$ \\
\hline \multicolumn{4}{|l|}{ Positive association expected: } \\
\hline Chocolate, sweets & $0.179^{*}$ & $0.310^{\star \star}$ & $0 \cdot 104$ \\
\hline Cakes, sweet biscuits, pastry & $0.306^{\star \star \star}$ & $0.401^{\star \star \star}$ & $0.251^{\star *}$ \\
\hline Chips, salty snacks & -0.036 & -0.010 & -0.061 \\
\hline Dishes with meat & 0.118 & 0.024 & 0.158 \\
\hline Jam & $0 \cdot 178^{*}$ & 0.197 & 0.161 \\
\hline Cheese & -0.039 & 0.024 & -0.080 \\
\hline \multicolumn{4}{|l|}{ Negative association expected: } \\
\hline Fruit & 0.006 & 0.126 & -0.059 \\
\hline Salad, raw vegetables & -0.094 & 0.087 & $-0.193^{\star}$ \\
\hline Boiled vegetables & -0.042 & -0.111 & 0.026 \\
\hline Potatoes & 0.031 & -0.019 & 0.052 \\
\hline Bread & 0.012 & 0.061 & -0.006 \\
\hline Dishes with fish & $-0 \cdot 204^{\star *}$ & -0.114 & $-0.253^{\star \star}$ \\
\hline \multicolumn{4}{|c|}{ Drinks, negative association expected: } \\
\hline Water & $-0.261^{\star \star \star}$ & -0.109 & $-0.360^{\star \star *}$ \\
\hline Tea & $-0.612^{\star \star *}$ & $-0.631^{\star \star \star}$ & $-0.599^{\star *}$ \\
\hline Juice & $-0.273^{\star \star \star}$ & $-0.220^{*}$ & $-0.311^{\star \star \star}$ \\
\hline Milk & -0.073 & $-0 \cdot 243^{\star}$ & 0.045 \\
\hline Lemonade & -0.053 & -0.146 & -0.008 \\
\hline Soft drinks & $-0.149^{*}$ & -0.078 & $-0 \cdot 207^{\star}$ \\
\hline Alcoholic beverages & 0.087 & 0.149 & 0.035 \\
\hline
\end{tabular}

${ }^{*} P<0.05,{ }^{* \star} P<0.01,{ }^{* * \star} P<0.001$.

†For details of subjects and procedures, see p. 262.

¥Spearman rank correlation, ordinal by ordinal, two-tailed significance.

$\S$ As some of the comparisons contained missing values, $n$ varied in these analyses from 200 to 205 . 
Table 5. Distribution of self-evaluated changes in intake of 'chocolate, sweets', 'cakes, sweet biscuits, pastry', 'jam', 'dishes with fish', and 'tea' in the four participant groups*

\begin{tabular}{|c|c|c|c|c|}
\hline & Abstainers ( $n 69)$ & Decreased ( $n$ 45) & Unchanged (n 51) & Increased ( $n$ 40) \\
\hline \multicolumn{5}{|l|}{ Chocolate, sweets: } \\
\hline Decreased intake (\%) & $17 \cdot 6$ & $20 \cdot 5$ & $8 \cdot 2$ & 2.5 \\
\hline Unchanged intake (\%) & $67 \cdot 6$ & $72 \cdot 7$ & $85 \cdot 7$ & 67.5 \\
\hline Increased intake (\%) & $14 \cdot 7$ & $6 \cdot 8$ & $6 \cdot 1$ & $30 \cdot 0$ \\
\hline All (\%) & $100 \cdot 0$ & $100 \cdot 0$ & $100 \cdot 0$ & $100 \cdot 0$ \\
\hline \multicolumn{5}{|c|}{ Cakes, sweet biscuits, pastry: } \\
\hline Decreased intake (\%) & $16 \cdot 4$ & 8.9 & $2 \cdot 0$ & $5 \cdot 1$ \\
\hline Unchanged intake (\%) & $79 \cdot 1$ & 84.4 & $89 \cdot 8$ & 61.5 \\
\hline Increased intake (\%) & 4.5 & $6 \cdot 7$ & $8 \cdot 2$ & $33 \cdot 3$ \\
\hline All (\%) & $100 \cdot 0$ & $100 \cdot 0$ & $100 \cdot 0$ & $100 \cdot 0$ \\
\hline \multicolumn{5}{|l|}{ Jam: } \\
\hline Decreased intake (\%) & 8.8 & 4.4 & $6 \cdot 1$ & 7.5 \\
\hline Unchanged intake (\%) & $88 \cdot 2$ & $91 \cdot 1$ & $91 \cdot 8$ & 67.5 \\
\hline Increased intake (\%) & 2.9 & 4.4 & $2 \cdot 0$ & $25 \cdot 0$ \\
\hline All (\%) & $100 \cdot 0$ & $100 \cdot 0$ & $100 \cdot 0$ & $100 \cdot 0$ \\
\hline \multicolumn{5}{|l|}{ Dishes with fish: } \\
\hline Decreased intake (\%) & 1.4 & $2 \cdot 2$ & $8 \cdot 2$ & 7.5 \\
\hline Unchanged intake (\%) & 85.5 & $91 \cdot 1$ & $91 \cdot 8$ & 87.5 \\
\hline Increased intake (\%) & $13 \cdot 0$ & 6.7 & 0.0 & $5 \cdot 0$ \\
\hline All (\%) & $100 \cdot 0$ & $100 \cdot 0$ & $100 \cdot 0$ & $100 \cdot 0$ \\
\hline \multicolumn{5}{|l|}{ Tea: } \\
\hline Decreased intake (\%) & 2.9 & $2 \cdot 2$ & $10 \cdot 2$ & $30 \cdot 0$ \\
\hline Unchanged intake (\%) & $29 \cdot 0$ & $51 \cdot 1$ & $87 \cdot 8$ & 67.5 \\
\hline Increased intake (\%) & $68 \cdot 1$ & $46 \cdot 7$ & $2 \cdot 0$ & 2.5 \\
\hline All (\%) & 100.0 & $100 \cdot 0$ & $100 \cdot 0$ & $100 \cdot 0$ \\
\hline
\end{tabular}

${ }^{*}$ For details of subjects and procedures, see p. 262.

biscuits and pastry' showed a positive association with change in coffee intake for both genders together. When the results were analysed for men only, the associations were even stronger, while the association with 'chocolate and sweets' was insignificant for the women. In addition, 'jam' had a positive relation with change in coffee intake, but was not significant in analyses of each gender separately.

Only the category 'dishes with fish' showed a significant negative association with change in coffee intake for the sample as a whole, but 'salad, raw vegetables' was negatively associated with coffee intake changes in the separate analyses of women. The other food categories did not show any significant associations with change in coffee intake.

Changes in the intake of tea, and to a lesser extent water, juice and soft drinks were significantly and inversely related to change in coffee intake. The inverse association between the change in coffee consumption and both water and soft drinks were significant among women, but not the men. For milk, an inverse association was found only among the men.

The use of Spearman rank correlation to examine the associations between change in coffee intake and change in diet gives little insight in the overall distribution of answers. Thus, crude cross-tables for the four significant dietary associations and tea, the most important substitute of liquid, are presented in Table 5.

Table 6 describes self-reported physical activity pattern during the intervention period compared with the participants' normal schedule. There was a significant negative association between change in coffee intake and change in physical activity level during the trial. Among the coffee abstainers, $14.5 \%$ reported that they had been more active than usual during the trial compared with none among those with increased coffee intake. In contrast, $10 \%$ of those with increased coffee intake had been less active, compared with $2.9 \%$ among the abstainers. We did not see any systematic changes in body weight between the groups (results not shown).

\section{Discussion}

Despite the fact that the participants in this trial were asked to stick to their habitual diet, a high proportion admitted to

Table 6. Self-reported physical activity pattern during the intervention compared with the participants' normal schedule* $\dagger$

\begin{tabular}{lccccr}
\hline & All $(n$ 205) & Abstainers $(n$ 69) & Decreased $(n$ 45) & Unchanged $(n$ 51) & Increased $(n$ 40) \\
\hline Less active (\%) & 6.3 & 2.9 & 6.7 & 7.8 & 10.0 \\
As normal (\%) & 82.0 & 82.6 & 80.0 & 78.4 & 90.0 \\
More active (\%) & 11.2 & 14.5 & 13.3 & 13.7 & 0.0 \\
All (\%) & 100.0 & 100.0 & 100.0 & 100.0 & 100.0 \\
\hline
\end{tabular}

* For details of subjects and procedures, see p. 262.

†Spearman rank correlation (ordinal by ordinal): $r-0.164, P<0.05$. 
having changed their diet during the intervention period. The participants did not only report compensations in fluid intake, but also changes in other food categories. These unwanted dietary changes went mainly in the directions expected from the cross-sectional studies with a more atherogenic diet among high coffee consumers. No significant associations were found in the opposite direction than expected.

As mentioned earlier, two main explanations may be offered if these associations are true: (1) a general lack of interest in a healthy lifestyle in high coffee consumers; or (2) coffee intake can alter food preferences through an unknown mechanism. In a controlled trial, the first explanation should be neutralized. The reported changes may also reflect methodologically induced bias, but we find it unlikely that allocation to a particular group would influence the response pattern. With the multiple statistical testing in the present study some spurious significant results can be expected, but since the results point in the same direction as cross-sectional findings we believe that several of these are true associations. We are therefore left with a number of systematic dietary changes that are unexplained.

As the main objective of the present trial was to study the effect of coffee on metabolic variables, the importance of maintaining a constant diet and lifestyle was stressed. Thus, the dietary changes reported here may possibly underestimate the effect of coffee on dietary habits, as participants are prone to answer according to the instructions.

It therefore appears that changes in coffee intake modify our food habits. To what extent this reflects a physiological phenomenon or cultural conventions about foods that go together with coffee cannot be answered by the present study. In favour of a physiological explanation is the tendency that smoking and physical inactivity also accompany this picture (Jacobsen \& Thelle, 1987; Solvoll et al. 1989; Puccio et al. 1990).

From a methodological point of view, the present study demonstrates the importance of monitoring behavioural changes in trials, particularly in unblinded trials with interventions affecting the daily way of living. Here, changes in one factor brought about other changes that could have consequences for the interpretation of the study outcome.

To our knowledge, modified food preferences associated with changes in coffee intake have never been described before. The mechanisms behind the associations between health-related behaviours should be elucidated further. They may be chains in behaviour, with either positive or negative health effects, where one behavioural change brings about another. This is a very complex relation to study, but may have implications for strategies in nutrition promotion and preventive medicine.

\section{Conclusions}

Induced changes in coffee intake seem to alter ad libitum intake of several foods. The recognized associations between health behaviours, here demonstrated for coffee intake, diet and physical activity, may have physiological explanations.

\section{Acknowledgements}

We thank Associate Professor H. Gjessing from the Section of Medical Statistics, University of Oslo, and Nibia Aires from the Institute of Cardiovascular Diseases, Sahlgrenska University Hospital for valuable statistical advice. The study was supported by grants from the Institute of Scientific Information on Coffee (ISIC) and the Physiological Effects of Coffee (PEC).

\section{References}

Andersen LF, Solvoll K \& Drevon CA (1996) Very-long-chain $n-3$ fatty acids as biomarkers for intake of fish and $n-3$ fatty acid concentrates. American Journal of Clinical Nutrition 64, 305-311.

Andersen LF, Solvoll K, Johansson LR, Salminen I, Aro A \& Drevon CA (1999) Evaluation of a food-frequency questionnaire with weighed records, fatty acids, and alphatocopherol in adipose tissue and serum. American Journal of Epidemiology 150, 75-87.

Christensen B, Mosdøl A, Rettersdøl L, Landaas S \& Thelle DS (2001) Abstention from filtered coffee reduces the levels of homocysteine and cholesterol - a randomized, controlled trial. American Journal of Clinical Nutrition 74, 302-307.

Haffner SM, Knapp JA, Stern MP, Hazuda HP, Rosenthal M \& Franco LJ (1985) Coffee consumption, diet, and lipids. American Journal of Epidemiology 122, 1-12.

Jacobsen BK \& Thelle DS (1987) The Tromso Heart Study: is coffee drinking an indicator of a life style with high risk for ischemic heart disease? Acta Medica Scandinavica 222, 215-221.

Nes M, Frost Andersen L, Solvoll K, Sandstad B, Hustvedt BE, Lovo A \& Drevon CA (1992) Accuracy of a quantitative food frequency questionnaire applied in elderly Norwegian women. European Journal of Clinical Nutrition 46, 809-821.

Puccio EM, McPhillips JB, Barrett-Connor E \& Ganiats TG (1990) Clustering of atherogenic behaviors in coffee drinkers. American Journal of Public Health 80, 1310-1313.

Solvoll K, Selmer R, Loken EB, Foss OP \& Trygg K (1989) Coffee, dietary habits, and serum cholesterol among men and women 35-49 years of age. American Journal of Epidemiology 129, $1277-1288$. 